\title{
Neck dissection complications
}

\author{
Complicações dos esvaziamentos cervicais
}

\author{
Rogério Aparecido Dedivitis ${ }^{1}$, André Vicente Guimarães ${ }^{2}$, Elio Gilberto Pfuetzenreiter Jr. ${ }^{3}$, Mario Augusto \\ Ferrari de Castro ${ }^{4}$
}

\begin{abstract}
Keywords:
intraoperative

complications,

postoperative

complications,

neck dissection.
\end{abstract}

Palavras-chave:

complicações

intra-operatórias, complicações

pós-operatórias, esvaziamento cervical.

\begin{abstract}
$\mathrm{B}$ (ND) for the treatment of patients with squamous cell carcinoma of the upper aerodigestive tract. Aim: To establish the incidence of complications of ND. Methods: A cross-sectional retrospective study of patient registries. ND with curative intention was evaluated in 480 patients with squamous cell carcinoma of the upper aerodigestive tract from January 1995 to December 2008 to identify perioperative complications. Results: Considering the total quantity of dissected neck sides, 413 radical ND and 295 selective ND were studied, of which 220 were supraomohyoid ND and 75 were jugular ND, totaling 708 sides. There were no deaths. The most frequent complication was marginal mandibular nerve injury (5.5\%), followed by accessory nerve injury (5.1\%). However, in 18 out of 21 cases this nerve was sacrificed for oncological completeness. Conclusions: There were no perioperative deaths. Nerves were the most commonly injured structures; the marginal mandibular branch is injured most (5.5\%).
\end{abstract}

\section{Resumo}

$\mathrm{D}$ evido à proximidade de estruturas vitais, certas complicações são inerentes ao esvaziamento cervical (EC) para o tratamento de pacientes portadores de carcinoma epidermoide das vias aerodigestivas superiores. Objetivo: Determinar a incidência de complicações dos EC. Métodos: Estudo retrospectivo transversal de avaliação de prontuários. Entre janeiro de 1995 e dezembro de 2008, foram avaliados EC com finalidade curativa em 480 pacientes portadores de carcinoma espinocelular das vias aerodigestivas superiores, sendo estudada a ocorrência de complicações perioperatórias. Resultados: Considerando o número total de lados esvaziados, foram estudados 413 EC radicais e 295 EC seletivos, dos quais 220 foram EC supraomo-hioideos e 75 foram EC jugulares, totalizando 708 lados avaliados. Não houve óbito. A complicação mais frequente foi a lesão do nervo mandibular marginal (5,5\%), seguida da lesão do nervo acessório (5,1\%), contudo, destes, em 18/21 casos o nervo foi sacrificado por razões de radicalidade oncológica. Conclusões: Não houve óbito no período peroperatório. As lesões mais comuns são nervosas, com maior incidência de lesão do nervo mandibular marginal (5,5\%).

\footnotetext{
${ }^{1}$ Professor Livre Docente pela Fundação Lusíada UNILUS, Médico.

${ }^{2}$ Doutor pelo Departamento de Cirurgia da Faculdade de Medicina da Universidade de São Paulo, São Paulo/SP, Assistente dos Serviços de Cirurgia de Cabeça e Pescoço do Hospital Ana Costa e da Irmandade da Santa Casa da Misericórdia de Santos, Santos/SP, Brasil.

${ }^{3}$ Mestre em Ciências pelo Curso de Pós-graduação do Hospital Heliópolis, São Paulo/SP. Professor do Departamento de Cirurgia da Fundação Lusíada UNILUS, Santos. Assistente dos Serviços de Cirurgia de Cabeça e Pescoço do Hospital Ana Costa e da Irmandade da Santa Casa da Misericórdia de Santos, Santos/SP, Brasil.

${ }^{4}$ Médico Residente de Cirurgia de Cabeça e Pescoço do Hospital Ana Costa, Santos. Professor do Departamento de Cirurgia da Fundação Lusíada UNILUS, Santos/SP, Brasil. Serviços de Cirurgia de Cabeça e Pescoço do Hospital Ana Costa e da Irmandade da Santa Casa da Misericórdia de Santos, Santos/SP, Brasil. Endereço para correspondência: Rogério A. Dedivitis - Rua Dr. Olinto Rodrigues Dantas 343 conj. 92 Santos SP 11050-220 Brasil. E-mail: dedivitis.hns@uol.com.br

Este artigo foi submetido no SGP (Sistema de Gestão de Publicações) da BJORL em 30 de dezembro de 2009. cod. 6857 Artigo aceito em 25 de fevereiro de 2010.
} 


\section{INTRODUÇÃO}

Esvaziamento cervical (EC) é um procedimento cirúrgico empregado para diagnóstico (estadiamento) e tratamento do câncer de cabeça e pescoço. O EC envolve a excisão de linfonodos de regiões específicas do pescoço com ou sem a remoção do músculo esternocleidomastoideo, veia jugular interna e nervo acessório. A exérese dos linfonodos cervicais não somente auxilia no estadiamento da neoplasia, como também trata a doença linfática e estabelece a necessidade de tratamento adjuvante, como a radioterapia. Devido à proximidade de estruturas vitais, certos riscos e complicações são inerentes a essa operação ${ }^{1}$. A prevenção, reconhecimento e tratamento das complicações precoces e tardias do EC são importantes na condução desses pacientes.

Desde a introdução do EC radical no início do Século 20, por $\mathrm{Crile}^{2}$, modificações têm sido propostas, notadamente a realização do esvaziamento funcional, por Suárez ${ }^{3}$, visando uma abordagem mais conservadora que permitisse a preservação de estruturas vitais do pescoço sem o comprometimento da radicalidade da remoção das estruturas linfáticas. A transição do EC radical para os seletivos reduziu os índices de complicações e morbidade, mantendo a eficácia cirúrgica e os princípios oncológicos ${ }^{4}$.

Como uma alternativa à ressecção cirúrgica primária, a quimiorradioterapia tem sido investigada como uma opção terapêutica para preservação de órgãos em pacientes com tumores avançados da cabeça e pescoço. A doença metastática cervical é um dos mais significativos fatores prognósticos, sendo geralmente menos responsiva que os tumores primários aos protocolos de preservação de órgão. Assim, para pacientes portadores de doença regional avançada, tem sido proposta a realização do EC planejado e de resgate após a quimiorradioterapia ${ }^{5}$, porém, com possibilidade de maior índice de complicações.

O objetivo desse estudo é determinar a incidência de complicações em pacientes portadores de carcinoma epidermoide das vias aerodigestivas superiores submetidos ao EC como modalidade de tratamento.

\section{MÉTODOS}

Entre janeiro de 1995 e dezembro de 2008, foram realizados EC com finalidade curativa em 480 pacientes portadores de carcinoma espinocelular das vias aerodigestivas superiores, sendo 434 do gênero masculino e 46 do feminino. Os pacientes foram avaliados retrospectivamente através do estudo transversal de seus prontuários. A idade média dos pacientes foi de 56 anos, variando de 33 a 75 anos. A Tabela 1 mostra a distribuição pelos sítios primários. Quando o sítio primário está na nasofaringe (linfoepitelioma), o tratamento primário indicado em nosso serviço é a associação de radioterapia e quimioterapia concomitante. Assim, todos os casos submetidos a EC para
Tabela 1. Distribuição dos pacientes conforme o sítio primário da doença.

\begin{tabular}{lll}
\hline $\begin{array}{l}\text { Momento do } \\
\text { esvaziamento }\end{array}$ & Sítio primário & $\begin{array}{l}\text { Número de } \\
\text { pacientes }\end{array}$ \\
\hline & Boca & 219 \\
Orofaringe & 52 \\
Laringe & 97 \\
Hipofaringe & 19 \\
Tratamento primário & Seios paranasais & 6 \\
& Pele & 2 \\
& Primário oculto & 41 \\
& Total & 436 \\
Após protocolo de preservação & Laringe & 10 \\
de órgão ou resgate de recidiva & Hipofaringe & 5 \\
de nasofaringe & Nasofaringe & 6 \\
& Total & 44 \\
\hline
\end{tabular}

tal sítio foram resgate de recidiva cervical, sendo estratificados juntos com os casos de protocolo de preservação de órgão. A Tabela 2 apresenta o tipo e o número de EC.

A indicação do EC dependeu do estadiamento cervical (N): EC seletivo foi realizado na ausência de doença evidente ou na vigência de doença cervical mínima (pescoço N1 em casos selecionados de câncer de boca); e EC radical clássico e radical modificado na vigência de adenopatia cervical clínica, sempre se tentando preservar as estruturas não linfáticas do pescoço (nervo acessório e veia jugular interna), desde que não houvesse comprometimento da radicalidade cirúrgica. EC bilateral foi indicado se houvesse comprovação ou suspeita de doença contralateral, sendo seletivo ou radical, de cada lado, conforme os critérios acima. Radioterapia adjuvante foi indicada em caso de metástase comprovada ao exame histopatológico ou de oportunidade por critérios de extensão ou agressividade do tumor primário. Radioterapia e quimioterapia concomitante adjuvantes foram indicadas na documentação de invasão extracapsular e em todos os casos N3.

Foi avaliada a ocorrência das seguintes complicações: deiscências de sutura (epidermólise; e deiscência

Tabela 2. Tipos de EC realizados.

\begin{tabular}{lc}
\hline \multicolumn{1}{c}{ Tipo de EC } & Número de pacientes \\
\hline Seletivo unilateral & 113 \\
Seletivo bilateral & 42 \\
Radical unilateral & 139 \\
Radical + seletivo contra-lateral & 98 \\
Radical bilateral & 88 \\
\hline
\end{tabular}


profunda); infecção /supuração; fístula quilosa; hemorragia (intra-operatória e pós-operatória); enfisema de subcutâneo; pneumotórax; fístulas salivares; estase venosa cefálica (manifestada por edema/ cianose faciais, sufusão/ edema conjuntivais; proptose; ingurgitamento venoso retinal; e convulsões/ depressão cardiorrespiratória); broncopneumonia e complicações pulmonares; lesões nervosas (nervos acessório, mandibular marginal, vago, hipoglosso, frênico e tronco simpático cervical); complicações incomuns (fístula artério-venosa, síndrome de secreção inapropriada do $\mathrm{ADH}$, acidente vascular cerebral, embolia aérea e cegueira); e mortalidade perioperatória.

\section{RESULTADOS}

Considerando o número total de lados esvaziados, foram estudados 413 EC radicais e 295 EC seletivos, dos quais 220 foram EC supraomo-hioideos e 75 foram EC jugulares. No total, foram 708 lados avaliados. A Tabela 3 apresenta a incidência das complicações encontrada. Não houve óbito no período perioperatório. A complicação mais frequente foi a lesão do nervo mandibular marginal, verificada em 39 lados esvaziados (5,5\%), seguida da lesão do nervo acessório, observada em 21 lados de 413 submetidos ao EC radical (5,1\%), contudo, destes, em 18 o nervo foi sacrificado por razões de radicalidade oncológica.

\section{DISCUSSÃO}

Apesar de o EC ser um procedimento tecnicamente bem estabelecido, é passível de complicações. Complicações intraoperatórias, como hemorragia, perda de ligadura venosa podendo levar a embolia gasosa, fístula quilosa por lesão do ducto torácico e arritmia por manipulação do bulbo carotídeo costumam ser bem manejadas, contudo, podem ser desastrosas ao paciente. Dissecção cuidadosa e ligadura dos vasos são extremamente importantes para evitar tanto hemorragia intraoperatória quanto pós-operatória. A formação de hematomas é prevenida pela cuidadosa hemostasia e pelo uso de drenos de sucção contínua ${ }^{6}$.

A radioterapia prévia tem impacto na cicatrização após o EC. Tal efeito é dose-dependente, já que doses maiores levam a fibrose mais extensa, hipóxia e diminuição da migração leucocitária7. Tivemos 43 casos com epidermólise, normalmente na intersecção em "T" dos retalhos, todos conduzidos de forma conservadora, contudo, houve adicionalmente 11 casos de deiscência profunda, dos quais cinco foram abordados com a rotação de retalho

Tabela 3. Complicações dos Esvaziamento Cervical (EC).

\begin{tabular}{|c|c|c|c|c|c|}
\hline Complicação & $\begin{array}{l}\text { EC seletivo unilateral } \\
\qquad(n=113)\end{array}$ & $\begin{array}{l}\text { EC seletivo bilateral } \\
\quad(n=42)\end{array}$ & $\begin{array}{l}\text { EC radical unilateral } \\
\qquad(n=139)\end{array}$ & $\begin{array}{c}\text { EC radical + seletivo } \\
(\mathrm{n}=98)\end{array}$ & $\begin{array}{l}\text { EC radical bilateral } \\
\qquad(\mathrm{n}=88)\end{array}$ \\
\hline Hemorragia intra-operatória & 0 & 0 & 0 & 0 & 0 \\
\hline Hemorragia pós-operatória & 0 & 0 & 1 & 0 & 0 \\
\hline Epidermólise & 0 & 0 & 15 & 13 & 15 \\
\hline Deiscência profunda & 0 & 0 & 3 & 4 & 4 \\
\hline Infecção da ferida & 0 & 0 & 1 & 0 & 1 \\
\hline Fístula quilosa & 0 & 0 & 0 & 1 & 2 \\
\hline Enfisema de subcutâneo & 0 & 0 & 0 & 0 & 0 \\
\hline Pneumotórax & 0 & 0 & 0 & 0 & 0 \\
\hline Fístula salivar & 0 & 0 & 0 & 0 & 0 \\
\hline Estase venosa cefálica & 0 & 0 & 0 & 0 & 6 \\
\hline Broncopneumonia & 0 & 1 & 1 & 2 & 2 \\
\hline Lesão do n. acessório & 0 & 0 & 9 & 7 & 5 \\
\hline $\begin{array}{l}\text { Lesão do n. mandibular } \\
\text { marginal }\end{array}$ & 6 & 5 & 8 & 11 & 9 \\
\hline Lesão do n. vago & 0 & 0 & 0 & 0 & 0 \\
\hline Lesão do n. hipoglosso & 0 & 0 & 0 & 1 & 2 \\
\hline Lesão do n. frênico & 0 & 0 & 0 & 0 & 1 \\
\hline Lesão do tronco simpático & 0 & 0 & 1 & 0 & 2 \\
\hline Complicações incomuns & 0 & 0 & 0 & 0 & 1 * \\
\hline Mortalidade perioperatória & 0 & 0 & 0 & 0 & 0 \\
\hline
\end{tabular}

$\left.{ }^{*}\right)$ cegueira bilateral 
pediculado. Em nossa casuística, não houve diferença significativa entre os casos previamente irradiados e aqueles com cirurgia primária.

O índice de infecção de parede é baixo e prevenido com o uso de antibióticos profiláticos por 24 horas. Esse baixo índice é atribuído à rica vascularização do pescoço e à natureza não contaminada do campo cervical. A contaminação cervical por ocasião de laringectomia ou de traqueostomia é considerada fator de maior índice de infecção, no entanto, a radioterapia prévia não apresenta impacto negativo ${ }^{8}$. Tivemos dois casos de infecção de parede, sendo um deles em paciente previamente irradiado. O uso de grampeador na confecção da neofaringe por ocasião da laringectomia total é um fator de proteção contra a contaminação cervical, prevenindo contra infecção do sítio cirúrgico?.

Fístula quilosa é uma complicação rara, ocorre de 1 a 2,5\% dos EC e a maioria incide no lado esquerdo. A porção mais acessível do ducto torácico localiza-se ao longo do aspecto medial da veia jugular interna e, como consequência, é a área onde a lesão mais frequentemente é causada. Se descoberta durante a cirurgia, manobras de pressão ventilatória positiva auxiliam na sua identificação e reparo ${ }^{10}$. Tivemos três casos após EC radical, sendo um abordado de maneira conservadora, com jejum e curativo compressivo e duas por meio de reoperação.

EC planejado ou de resgate após protocolo de preservação de órgão associa-se com o aumento da incidência de complicações, como deiscência e necrose de retalhos, principalmente quando se realiza procedimento em faringe e laringe, quando ocorre maior chance de fístula faringocutânea associada, podendo levar a um maior tempo de hospitalização e a procedimentos cirúrgicos adicionais ${ }^{6,11}$. Tivemos um caso de hemorragia pós-operatória, sendo o paciente reoperado, com evacuação do hematoma, irrigação dos retalhos e ligadura de uma tributária da jugular interna, cuja ligadura havia sido perdida.

Edema facial ocorre comumente, como consequência do comprometimento do retorno venoso após EC radical bilateral, podendo raramente ser maciço e acompanhado de edema cerebral e até óbito. Como rotina, tentamos preservar pelo menos uma das jugulares internas, mesmo assim, observamos tal complicação em seis de nossos casos de pacientes submetidos ao EC radical bilateral, contudo, sem complicações cerebrais.

Lesão de estrutura nervosa durante o EC não é incomum e pode levar tanto a déficit funcional como a síndrome dolorosa. A incidência costuma ser baixa quando se realiza EC funcional: 1,68\% de lesão do nervo acessório; $1,26 \%$ do mandibular marginal; $0,56 \%$ do hipoglosso; e $0,42 \%$ do simpático cervical, em casuística de 442 pacientes $^{12}$.

Lesão do mandibular marginal ocorre mais comumente por ocasião da elevação do retalho superior ou durante a dissecção do nível I (triângulo submentomandibular), podendo causar disfunção dos depressores do lábio inferior. Em estudo de 66 pacientes submetidos ao EC, lesão foi encontrada em 18\% dos pacientes e 23\% dos pescoços, resultando em assimetria ao sorriso, contudo, sem sequela grave ${ }^{13}$.

O nervo vago pode ser lesado por ocasião da ligadura da veia jugular interna durante o EC radical. Quando a lesão ocorre abaixo do gânglio nodoso, resulta em paralisia de prega vocal; se for acima, associa-se também com disfagia e aspiração ${ }^{14}$. É complicação rara e não a verificamos em nossa casuística. Lesão no frênico é incomum e, muitas vezes, não reconhecida, podendo resultar em atelectasias e infiltrado pulmonar ${ }^{15}$. Também não a observamos.

Com relação ao nervo acessório, mesmo quando é preservado, sua esqueletização resulta em desvascularização e a síndrome do ombro doloroso, não sendo tão incomum e tendo impacto na qualidade de vida do paciente. É essencial a familiaridade com a anatomia do triângulo posterior do pescoço. Em caso de transecção ou sacrifício por razões oncológicas, o reparo por meio de anastomose ou enxerto tem mostrado benefício. Por meio da aplicação de questionário específico, em 65 pacientes que participaram de um estudo, com um período de seguimento superior a 1,6 anos, 23\% negaram disfunção do ombro, enquanto 54\% referiram disfunção discreta, 15\% moderada e $8 \%$ grave $^{16}$.

A síndrome de Bernard-Horner ou paresia óculosimpática (blefaroptose, enoftalmo e miose) é causada por lesão do simpático cervical, estrutura localizada posterior e profundamente à bainha carotídea e que pode ser lesada durante o EC radical ${ }^{17}$, como ocorreu em três lados esvaziados em nossa casuística.

Dentre as complicações raramente descritas na literatura, a cegueira é uma das mais catastróficas, com 13 casos relatados $^{18}$. Pode resultar de infarto cortical, neuropatia óptica isquêmica posterior ou oclusão carotídea bilateral, sendo anemia, hipotensão e doença vascular pré-existente fatores de risco ${ }^{6}$. Tivemos um caso após EC radical bilateral, sem encontrar-se causa aparente.

As indicações de EC radical bilateral simultâneo continuam controversas. Na análise de 193 casos, tal procedimento apresentou alta morbidade, recomendando-se sua contra-indicação enquanto procedimento eletivo ${ }^{19}$. Em nosso estudo, também encontramos maiores índices de complicações nesse grupo de pacientes, porém, não obtivemos mortalidade peroperatória.

Com a progressiva popularização, a partir da década de 1990, da quimiorradioterapia com a finalidade de preservação de órgão e sendo a metástase cervical um significado fator prognóstico independente, tem-se indicado o EC com finalidade de resgate ou planejado após tal tratamento conservador. Entretanto, os índices de 
complicações de parede quando o EC é realizado entre cinco e 17 semanas após o protocolo são da ordem de $10 \%$, com necessidade, em $6 \%$, do fechamento a custo de retalho pediculado ${ }^{20}$. Tem sido proposta a opção de realizar-se o EC previamente à quimiorradioterapia, com índice de complicações de $2.5 \%{ }^{21}$.

\section{CONCLUSÕES}

Não houve óbito no período perioperatório. As lesões mais comuns são nervosas, com maior incidência de lesão do nervo mandibular marginal (5,5\%).

\section{REFERÊNCIAS BIBLIOGRÁFICAS}

1. Smullen JL, Lejeune FE. Complications of neck dissection. J La State Med Soc.1999;151(11):544-7.

2. Crile G. Excision of cancer of the head and neck. With special reference to the plan of dissection based on 132 patients. JAMA. 1906; 47:1780-6.

3. Suarez O. El problema de las metastasis linfáticas y alejadas del cáncer de laringe e hipofaringe. Rev Otorrinolaringol.1963;23:83-99.

4. Ferlito A, Gavilán J, Buckley JG, Shaha AR, Miodonski AJ, Rinaldo A. Functional neck dissection: fact and fiction. Head Neck.2001;23:804-8.

5. Wang SJ, Wang MB, Yip H, Calcaterra TC. Combined radiotherapy with planned neck dissection for small head and neck cancers with advanced cervical metastases. Laryngoscope.2000;110(11):1794-7.

6. Genden EM, Ferlito A, Shaha AR, Talmi YP, Robbins KT, Rhys-Evans $\mathrm{PH}$, et. al. Complications of neck dissection. Acta Otolaryngol. 2003;123(7):795-801.

7. Mathes SJ, Alexander J. Radiation injury. Surg Oncol Clin N Am. 1996;5(4):809-24
8. Coskun H, Erisen L, Basut O. Factors affecting wound infection rates in head and neck surgery. Otolaryngol Head Neck Surg.2000;123(3):32833.

9. Dedivitis RA, Guimarães AV. Use of stapler for pharyngeal closure after total laryngectomy. Acta Cir Bras.2004;19(1):66-9.

10. de Gier HH, Balm AJ, Bruning PF, Gregor RT, Hilgers FJ. Systematic approach to the treatment of chylous leakage after neck dissection. Head Neck.1996;18(4):347-51.

11. Dedivitis RA, Ribeiro KC, Castro MA, Nascimento PC. Pharyngocutaneous fistula following total laryngectomy. Acta Otorhinolaryngol Ital.2007;27(1):2-5.

12. Prim MP, De Diego JI, Verdaguer JM, Sastre N, Rabanal I. Neurological complications following functional neck dissection. Eur Arch Otorhinolaryngol.2006;263(5):473-6.

13. Batstone MD, Scott B, Lowe D, Rogers SN. Marginal mandibular nerve injury during neck dissection and its impact on patient perception of appearance. Head Neck.2009;31(5):673-8

14. Cece JA, Lawson W, Biller HF, Eden AR, Parisier SC. Complications in the management of large glomus jugulare tumors. Laryngoscope. 1987;97(2):152-7.

15. de Jong AA, Manni JJ. Phrenic nerve paralysis following neck dissection. Eur Arch Otorhinolaryngol. 1991;248(3):132-4

16. Carr SD, Bowyer D, Cox G. Upper limb dysfunction following selective neck dissection: a retrospective questionnaire study. Head Neck. 2009;31(6):789-92.

17. Bucci T, Califano L. Bernard-Horners syndrome: unusual complication after neck dissection. J Oral Maxillofac Surg.2008;66(4):833.

18. Worrell L, Rowe M, Petti G. Amaurosis: a complication of bilateral radical neck dissection. Am J Otolaryngol.2002;23(1):56-9.

19. Magrin J, Kowalski L. Bilateral radical neck dissection: results in 193 cases. J Surg Oncol.2000;75(4):232-40.

20. Ha PK, Couch ME, Tufano RP, Koch WM, Califano JA. Short hospital stay after neck dissection. Otolaryngol Head Neck Surg. 2005;133(5):677-80.

21. Prades JM, Timoshenko AP, Schmitt TH, Delolme MP, Francoz M, Martin C, et al. Planned neck dissection before combined chemoradiation for pyriform sinus carcinoma. Acta Otolaryngol.2008;128(3):324-8. 